\title{
Aplicabilidade dos campos 490 e 800-830 do Formato MARC 21 para Dados Bibliográficos
}

\author{
Márcia Carvalho Rodrigues \\ Mestre em letras, cultura e regionalidade pela Universidade \\ de Caxias do Sul (UCS), Caxias do Sul, RS - Brasil. \\ E-mail: mcrodrig@ucs.br
}

\section{Marcelo Votto Teixeira}

Bacharel em biblioteconomia pela Universidade Federal do Rio Grande (FURG). Especialista em didática e metologia para o ensino superior pelo Centro Universitário Anhanguera - Porto.

E-mail:mvteixeira@ucs.br

\section{Resumo}

O texto traz o histórico e a evolução dos campos 4XX Séries do Formato MARC 21 para Dados Bibliográficos. Aborda a recente mudança que tornou obsoleto 0 campo 440 em favor dos campos 490 e 800-830. Ilustra a aplicação dos campos 490 e 800-830 em registros bibliográficos por meio de exemplos, relacionando-os com as regras do Código de Catalogação Anglo-Americano, $2^{\mathrm{a}}$ edição e descreve o impacto desta mudança nos catálogos bibliográficos.

\section{Palavras-chave}

Formato MARC 21 para Dados Bibliográficos. Série. Catalogação.

\section{Applicability of the fields 490 and $800-830$ of the MARC 21 Format for Bibliographic Data}

\begin{abstract}
This text presents the history and evolution of the fields $4 X X$ - Series statement fields of the MARC 21 Format for Bibliographic Data. Recent change is discussed about the field 440 which was substituted by the fields 490 and 800-830. The application of the fields 490 and 800-830 is illustrated in the bibliographic records by examples mentioning their relation to the norms of the AngloAmerican Cataloguing Rules, $2^{\text {nd }}$ edition, describing the impact of this change on bibliographic catalogs.
\end{abstract}

\section{Keywords}

MARC 21 Format for Bibliographic Data. Series. Cataloging.

Ci. Inf., Brasília, DF, v. 39 n. 3, p.47-60, set./dez., 2010

\section{INTRODUÇÃO}

A padronização dos registros bibliográficos na catalogação é um subsídio fornecido aos profissionais para que possam unificar informações e facilmente transportá-las pelas redes de computadores. É uma tarefa em constante aprimoramento e dela depende não apenas o conhecimento de números, símbolos e suas representações dentro de um contexto sistematizado, mas o acompanhamento das mudanças tecnológicas e as consequentes adequações desses padrões a novos recursos informacionais.

Um dos padrões mais difundidos mundialmente é o MARC - MAchine-Readable Cataloging (Catalogação Legível por Máquina), criado na década de 1960 pela Biblioteca do Congresso norte-americano para atender sua própria demanda e necessidade de padronização de registros bibliográficos. O MARC é um formato de intercâmbio de registros legíveis por máquina e tem a função de promover a comunicação da informação, evitando a duplicação de esforços por meio do intercâmbio de registros bibliográficos. Neste trabalho serão abordados o MARC 21 Bibliográfico, cujo objetivo consiste na padronização do armazenamento de informações bibliográficas sobre recursos informacionais; e o MARC 21 Autoridade, cujo objetivo está focado na disponibilização de formas autorizadas de nomes e assuntos para serem usadas nos registros bibliográficos e no fornecimento de pontos de acesso nos catálogos, por intermédio da padronização dos nomes.

Observa-se que no Brasil, mesmo passados 50 anos da criação do formato MARC, ainda há pouca discussão sobre o padrão. Destaca-se também a ausência de posicionamento das entidades nacionais responsáveis pela catalogação ante as alterações realizadas no formato MARC e que diretamente afetam regras da $2^{a}$ edição do Código de Catalogação Anglo-Americano (AACR2), e, consequentemente, catálogos de bibliotecas de todo o país. 
Neste artigo será abordada a recente mudança ocorrida nos campos de representação de série em registros bibliográficos. A mudança nos campos 440, 490 e 800-830 afetará diretamente os catálogos de bibliotecas do mundo inteiro. Catálogos que ainda trabalham com o campo 440 terão incompatibilidade na importação de registros bibliográficos advindos de catálogos que já trabalham com o campo 490 e 800-830. Além do que, softwares gerenciadores de sistema de bibliotecas não serão capazes de migrar o campo 440 automaticamente para os campos 490 e 800-830, pois existe a necessidade de padronização dos campos 800-830 no MARC Autoridades. Desta forma, o trabalho apresentado busca elucidar a aplicação dos campos 490 e 800-830 em registros bibliográficos nos exemplos, relacionando-os com as regras do AACR2.

\section{O formato MARC 21 Bibliográfico}

Quando a Biblioteca do Congresso norte-americano começou a utilizar computadores na década de 1960, houve a necessidade de planejar um formato para registros bibliográficos - assim surgiu o formato MARC, que utiliza um sistema de números, letras e símbolos dentro do registro bibliográfico para indicar diferentes tipos de informação. $\mathrm{O}$ formato original LC MARC evoluiu para o MARC $21 \mathrm{e}$ tornou-se o padrão utilizado pela maioria dos softwares de biblioteca no mundo.

MARC, portanto, é a sigla de MAchine-Readable Cataloging (Catalogação Legível por Máquina), um conjunto de cinco formatos que servem de ferramenta ao universo bibliográfico, a saber:

- MARC 21 Format for Bibliographic Data (Formato MARC 21 para Dados Bibliográficos);

- MARC 21 Format for Authority Data (Formato MARC 21 para Dados de Autoridade);

- MARC 21 Format for Holdings Data (Formato MARC 21 para Dados de Coleção);

- MARC 21 Format for Classification Data (Formato MARC 21 para Dados de Classificação);
- MARC 21 Format for Community Information (Formato MARC 21 para Informação Comunitária).

No Brasil, o formato MARC teve sua introdução na década de 70 com projetos paralelos ou, como denominado na época, projetos de formato MARC compatíveis. Entre os projetos, destacam-se o formato CALCO da Fundação Getúlio Vargas e o Formato IBICT do Instituto Brasileiro de Informação em Ciência e Tecnologia.

Ao longo do desenvolvimento dos projetos e a dificuldade de ampliação para os países da América Latina a fim de formar uma rede integrada de catalogação, dificuldade esta apresentada principalmente pelo fato de, na época, o Brasil ser o único país com um programa de catalogação atuante, as agências catalográficas brasileiras ingressaram na Online Computer Library Center (OCLC), o que foi fator preponderante para o avanço e domínio do formato MARC 21 (na época, USMARC) na descrição dos registros nas bibliotecas brasileiras.

Atualmente, as entidades responsáveis pela manutenção e atualização dos formatos MARC 21 são a Biblioteca do Congresso norte-americano e a Biblioteca Nacional do Canadá.

\section{Evolução dos campos 4XX - Série}

No Formato MARC 21 para Dados Bibliográficos, os campos 4XX designam as entradas de autor/ título ou título usado como uma entrada de série em registros bibliográficos. Até 1999, o MARC previa os seguintes campos para registro de série:

400 Series statement/ Added entry - Personal name (Título de série/Entrada secundária - Nome pessoal);

410 Series statement/Added entry - Corporate name (Título de série/Entrada secundária - Entidade coletiva);

411 Series statement/ Added entry - Meeting name (Título de série/Entrada secundária - Evento);

440 Series statement/Added entry - Title (Título de série/Entrada secundária - Título);

490 Series statement (Título de série relacionado). 
Aplicabilidade dos campos 490 e 800-830 do Formato MARC 21 para Dados Bibliográficos

O conteúdo dos campos 400-440 servia tanto para indicar um título de série quanto uma entrada secundária de série (autoridade controlada), já que sua função incluía possibilitar o registro do título de uma série quando esta se apresenta da mesma maneira tanto na obra quanto na sua forma autorizada.

Já o campo 490 deveria ser utilizado para registrar um título de série não controlado ou que apresentará, na sua forma controlada, uma entrada diferente daquela descrita no campo 490. Assim, a forma controlada da série deveria ser indicada nos campos 800-830 - Series added entry fields (Entradas secundárias de série), ou seja, os campos 800-830 somente seriam utilizados se houvesse um título de série registrado no campo 490.

Com a introdução da $2^{\mathrm{a}}$. edição do Código de Catalogação Anglo-Americano (AACR2), os campos 400, 410 e 411 deixaram de ser utilizados, permanecendo somente os campos 440 e 490 para designar títulos de série (no MARC 21 esses campos são descritos no Apêndice H - Local Data Elements).

A partir de outubro de 2008, o campo 440 tornouse obsoleto. Permanecem, atualmente, apenas os campos 490 e 800-830. Tecnicamente, portanto, o 440 deve deixar de ser utilizado pelas instituições ao redor do mundo que utilizam o padrão MARC para registros bibliográficos.

De acordo com o Apêndice H - Local data elements do MARC 21, a substituição do campo 440 ocorreu visando a simplificar a área da série, já que anteriormente o MARC previa duas formas diferentes de registro: 440 e 490, o que possibilitava a duplicidade do registro de séries.

\section{Os trâmites da mudança}

A entidade responsável pela alteração no uso do campo 440 e a utilização dos campos 490 e 800830 é o Program for Cooperative Cataloging (Programa de Catalogação Cooperativa - PCC), vinculado à Biblioteca do Congresso. Tal afirmativa constata-se nos documentos apresentados pelo programa junto ao Comitê Consultivo do MARC.
A intenção da mudança foi resolver um problema de longa data que o campo 440 gerava aos consultores e usuários do MARC, pois este campo é tanto uma descrição quanto um ponto de acesso controlado. Já separando as duas funções, os catalogadores teriam uma descrição mais confiável e um fácil controle por meio da distinção dos campos.

O primeiro documento apresentado oficialmente foi uma proposta para análise, em 7 de dezembro de 2007, sob o registro MARC Discussion Paper $n$. 2008-DP02, e possuía a seguinte ementa:

Este documento sugere tornar o campo 440 obsoleto em favor do uso dos campos 490 (Série relacionada) e 8XX Entradas secundárias de série. Isto simplificaria a prática e a necessidade dos sistemas procurarem pelo cabeçalho de série autorizado em vários lugares dos registros.

A partir desse documento torna-se claro o objetivo de simplificar o controle dos campos ligados à série. Assim, em 14 de fevereiro de 2008, o Comitê Consultivo do MARC emitiu uma resposta em relação ao documento enviado para análise:

Lidar com os dados antigos foi discutido em detalhes. Embora alguns participantes estivessem preocupados com o fato de que os vendedores de seus sistemas

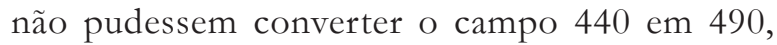
apontou-se que, baseado nos princípios do MARC, dados obsoletos estão autorizados a permanecer em registros válidos. Os participantes também expressaram preocupação sobre tornar o campo 440 obsoleto, o que causaria a repetição dos dados nos campos 490 e 800-830. No entanto, um dos membros do Comitê salientou que a economia de espaço de armazenamento dos sistemas não é mais uma preocupação para a maioria dos sistemas no ambiente computacional de hoje. Houve também a discussão de que tornar o campo 440 obsoleto deveria ser tratado como uma questão de implementação política, ao invés de uma mudança no formato bibliográfico. Os participantes em geral concordaram em definir o subcampo \$x (ISSN) nos campos 800-830 para melhorar a indexação de entradas secundárias de série. Foi mencionado que incluir uma designação para outros identificadores, como o ISBN, poderia ser benéfica. Sugeriu-se dividir a proposta em duas 
partes: uma para tornar o campo 440 obsoleto e outra para definir o subcampo \$x nos campos 800-830.

Nesse primeiro entendimento do Comitê Consultivo já se percebe a visão estratégica da entidade perante o impacto que as alterações gerariam para os profissionais e para as empresas desenvolvedoras de softwares que trabalham com o formato MARC. Outros três pontos pertinentes a esse documento são: (1) a confirmação da impossibilidade de haver transferência dos dados contidos no campo 440 para o campo 490, bem como (2) a possibilidade de que caso o campo 440 torne-se obsoleto não precise ser extinto das bases, liberando as entidades catalogadoras para uma transferência gradual dos campos e (3) a definição dos campos 800-830 para a inserção dos dados de forma controlada.

Após o envio para o comitê consultivo do MARC e a verificação da possibilidade de efetuar as alterações pretendidas, o PCC enviou em 6 de junho de 2008 duas propostas de alterações do MARC Bibliográfico, a MARC Proposal n. 2008-06 e MARC Proposal n. 2008-07.

A MARC Proposaln. 2008-06, cuja responsabilidade foi assumida pela Cooperative Online Serials (Programa de Cooperação Online de Publicações Seriadas CONSER), grupo subordinado ao PCC, propunha o seguinte:

Este documento propõe um mecanismo para indicar quais números de uma série, monografia multiparte, conjunto de mapas, etc. manifestam um título específico de série. Recomenda a definição do subcampo $\$ 3$ (Especificação do material) para designar a parte do recurso para a qual a informação se aplica nos campos de série 8XX e no campo 490. Além disso, propõe o acréscimo de um subcampo $\$ \mathrm{x}$ para ISSN nos campos de série 8XX.

Nessa primeira proposta a CONSER prepara os campos 490 e 8XX para receberem os dados das séries. Assim, é acrescentado o subcampo $\$ 3$ (Especificação do material) para ser utilizado pelos campos 490 e 8XX e que servirá para representar especificações do material descrito, e o subcampo \$x (ISSN) para o campo 8XX.
Após aprovada em 2 de outubro de 2008, a $M A R C$ Proposal n. 2008-06 evidencia a mudança que estava por vir. Isso é constatado na discussão que definiria a utilização do subcampo \$x (ISSN) pelo campo 8XX, em que já se menciona a MARC Proposal n. 2008-07:

A MARC Proposal 2008-07 propõe tornar obsoleto o campo $440 \mathrm{em}$ favor do campo 490. Atualmente, no campo 440, números de ISSN podem ser registrados no subcampo \$x. Por fornecer um ponto de acesso útil para as séries, foi proposto adicionar o subcampo \$x nos campos de entrada secundária de série 8XX. Os comentários ao Discussion Paper No. 2008-DP02 indicaram que uma alternativa para a definição de um subcampo para estes códigos no 8XX seria expandir as definições do 022 para fornecer um ISSN que identifique uma série. No entanto, essa opção não está sendo considerada, pois a Rede ISSN não permite a repetição do campo 022 e múltiplos campos 022 são rejeitados para o processamento no banco de dados do ISSN. Foi salientado que fornecer o ISSN em um campo 8XX poderia facilitar a pesquisa em outros sistemas, como resolvedores de links.

Apresentada juntamente com a MARC Proposal $n$. 2008-06, a MARC Proposal n. 2008-07, agora com a autoria do PCC, propõe que o campo 440 fique obsoleto em favor do uso dos campos 490 e 8XX e a alteração da função do Indicador 1 do campo 490. Assim, é definida a proposta na da seguinte ementa:

Este documento propõe tornar o campo 440 obsoleto em favor do uso do 490 (Série relacionada) e dos campos de entrada secundária de série 8XX para séries relacionadas. Isso simplifica a prática e a necessidade dos sistemas procurarem pelo cabeçalho autorizado de série em vários lugares nos registros. $\mathrm{O}$ documento também propõe mudanças na definição do valor 1 do primeiro indicador do campo 490.

Antes da discussão sobre as alterações propostas, relacionam-se os documentos já analisados que tratam sobre o mesmo assunto:

O Discussion Paper 2008-DP02 descreve como tornar o campo 440 (Série relacionada / Entrada secundária) 
Aplicabilidade dos campos 490 e 800-830 do Formato MARC 21 para Dados Bibliográficos

obsoleto. A intenção da proposta é resolver um problema de longa data relacionado ao fato do campo 440 ser tanto um campo descritivo quanto um ponto de acesso controlado. Separar as duas funções resultará em uma descrição mais confiável e de fácil manutenção ao longo do tempo através do controle de autoridade. O Comitê Consultivo do MARC discutiu o documento durante as reuniões de inverno em janeiro de 2008, e muitos participantes sentiram que lidar com dados antigos e com a repetição dos dados nos campos 440 e 490 eram questões que precisavam ser discutidas. Foi sugerido que tornar o campo 440 poderia ser tratado como uma questão de implementação política, ao invés de uma mudança no formato bibliográfico. O documento também discutiu o acréscimo do subcampo \$x para o ISSN (e talvez subcampos adicionais para outros identificadores padrão) nos campos 8XX, uma vez que este subcampo só está disponível nos campos 440 e 490. O Comitê solicitou que duas propostas fossem consideradas separadamente para tornar o campo 440 obsoleto e acrescentar subcampos aos campos 8XX; a proposta de acrescentar subcampos nos campos 8XX é a MARC Proposal No. 2008-06.

Ao discutir a modificação do campo 440 para condição de campo obsoleto, os membros do Conselho Consultivo do MARC retomam as posições já apresentadas na MARC Discussion Paper n. 2008-DP02 ao publicar que:

Alguns comentários sobre o 2008-DP02 expressaram preocupação sobre a repetição de dados nos campos 490 e 8 XX. Os benefícios de um processo de decisão simplificado, sem um impacto adverso sobre os sistemas locais ou a OCLC podem compensar as consequências mínimas da repetição de campos. A tomada de decisão simplificada também poderia auxiliar na formação de novos funcionários e reforçar fluxos de trabalho em bibliotecas. Havia também preocupações de que alguns sistemas locais não seriam capazes de converter o campo $440 \mathrm{em}$ 490. Por outro lado, salientou-se que, com base nos princípios do MARC, campos de dados obsoletos estão autorizados a permanecer nos registros. $\mathrm{O}$ documento de discussão presumiu que a OCLC investigará mudanças nos registros existentes no WorldCat para mover os dados do campo 440 para os campos 490 e $8 \mathrm{XX}$ se a proposta for aprovada. Isto apoiará a capacidade de controlar todos os cabeçalhos de série nos registros bibliográficos do WorldCat. Os parâmetros para selecionar quais registros mudam automaticamente, lidando com as diferenças na utilização de indicadores de não-arquivamento nos campos 440 e 830 , e eventuais conflitos com as orientações PCC existentes ainda precisam ser discutidas com a OCLC.

Também se verifica que o Conselho já designou a OCLC para realizar a transferência do campo 440 dos registros existentes na base de dados do WorldCat.

Sendo assim, a MARC Proposal n. 2008-07, também aprovada em 2 de outubro de 2008, define e direciona os catalogadores para a nova forma de proceder com relação ao registro das séries. De forma geral, resumindo consideravelmente todo o conteúdo analisado e aprovado, a representação das séries deverá ocorrer nos seguintes campos:

Campo descritivo:

490 Series statement (Título de série relacionado).

Campo controlado:

800 Series added entry - Personal name (Entrada secundária de série - Nome pessoal);

810 Series added entry - Corporate name (Entrada secundária de série - Entidade coletiva);

811 Series added entry - Meeting name (Entrada secundária de série - Evento);

830 Series added entry - Uniform title (Entrada secundária de série - Título uniforme).

\section{Campo 490 - Título de série relacionado: aplicação}

O campo 490 contém um título de série para o qual não foi gerada uma entrada secundária, ou para o qual a entrada secundária é uma autoridade controlada diferente da descrita no campo 490.

Diferentemente do campo 440, o campo 490 não serve como uma entrada secundária de série. Quando 
o campo 490 é utilizado e a instituição catalogadora deseja cadastrar uma entrada secundária (autoridade controlada), deverão ser utilizados os campos 490 e sua entrada secundária correspondente (campos 800-830), cadastrados separadamente.

Assim, o campo 490 é definido no MARC 21 como um título de série não relacionado e é utilizado para transcrever um título de série exatamente como este aparece no item que se está descrevendo.

O campo 490 é repetitivo (R) e inclui os seguintes elementos:

Indicadores:

1 Especifica se o título da série está relacionado, ou seja, se haverá ou não uma entrada secundária:

0 título não relacionado (não haverá entrada secundária);

1 título relacionado (haverá entrada secundária).

2 Indefinido (\#).

Subcampos:

\$a Título da série $(\mathrm{R})$;

\$1 Número de chamada da Library of Congress (NR);

\$v Volume ou número sequencial da série (R);

\$ $\mathrm{ISSN}(\mathrm{R})$;

\$3 Material especificado (NR);

\$6 Ligação (NR);

\$8 Campo de ligação e número sequencial (R).

Campos 800-830 - Entrada secundária de série: aplicação

Os campos 800-830 contêm um nome/título ou um título usado como uma entrada secundária de série quando o título de série está descrito no campo
490, ou no campo 500 (Notas gerais) e este exige uma entrada secundária no registro bibliográfico. Os campos 800-830 nunca são utilizados quando há título de série no campo 440, haja vista que este campo abrange as funções de título de série e entrada secundária de série.

Os campos 800-830 são definidos, portanto, como títulos relacionados, ou seja, são autoridades controladas e deverão ter suas entradas estabelecidas a partir do catálogo de autoridades da instituição. As entradas secundárias de série poderão ser definidas a partir dos seguintes campos:

800 Entrada secundária de série - Nome pessoal;

810 Entrada secundária de série - Entidade coletiva;

811 Entrada secundária de série - Evento;

830 Entrada secundária de série - Título uniforme;

840 Entrada secundária de série - Título ${ }^{1}$ [obsoleto].

800 Entrada secundária de série - Nome pessoal

O campo 800 é definido como uma entrada secundária de série na qual a entrada de autor é um nome pessoal. É utilizado sempre que o título de série descrito no campo 490 difere da forma controlada.

O uso do campo 800 geralmente se justifica pela presença de um 490 ('Título de série), ou um 500 (Notas gerais) relativo à série. Para reproduções, poderá ainda ser justificado por um título de série no subcampo \$f do campo 533 (Notas de reprodução).

O campo 800 é repetitivo $(\mathrm{R})$ e inclui os seguintes elementos:

\footnotetext{
${ }^{1}$ O campo 840 tornou-se obsoleto em 1980 . A partir de então de-
cidiu-se utilizar o campo 830 tanto para títulos uniformes quanto
para títulos convencionais que não têm entrada subordinada a no-
mes. O campo 840 continha os seguintes indicadores: $1^{\circ}$ indicador:
indefinido (\#), $2^{\circ}$ indicador: $0-9$ (número de caracteres a desprezar).
Os subcampos eram $\$$ a (Título), \$h (Meio [DGM]) e \$v (Volume
ou número).

${ }^{1}$ O campo 840 tornou-se obsoleto em 1980 . A partir de então de-
cidiu-se utilizar o campo 830 tanto para títulos uniformes quanto
para títulos convencionais que não têm entrada subordinada a no-
mes. O campo 840 continha os seguintes indicadores: $1^{\circ}$ indicador:
indefinido (\#), $2^{\circ}$ indicador: $0-9$ (número de caracteres a desprezar).
Os subcampos eram $\$ a$ (Título), $\$$ h (Meio [DGM]) e \$v (Volume
ou número).

${ }^{1}$ O campo 840 tornou-se obsoleto em 1980 . A partir de então de-
cidiu-se utilizar o campo 830 tanto para títulos uniformes quanto
para títulos convencionais que não têm entrada subordinada a no-
mes. O campo 840 continha os seguintes indicadores: $1^{\circ}$ indicador:
indefinido (\#), $2^{\circ}$ indicador: $0-9$ (número de caracteres a desprezar).
Os subcampos eram $\$ a$ (Título), $\$$ h (Meio [DGM]) e \$v (Volume
ou número).

${ }^{1}$ O campo 840 tornou-se obsoleto em 1980 . A partir de então de-
cidiu-se utilizar o campo 830 tanto para títulos uniformes quanto
para títulos convencionais que não têm entrada subordinada a no-
mes. O campo 840 continha os seguintes indicadores: $1^{\circ}$ indicador:
indefinido (\#), $2^{\circ}$ indicador: $0-9$ (número de caracteres a desprezar).
Os subcampos eram $\$$ a (Título), \$h (Meio [DGM]) e \$V (Volume
ou número).

${ }^{1}$ O campo 840 tornou-se obsoleto em 1980 . A partir de então de-
cidiu-se utilizar o campo 830 tanto para títulos uniformes quanto
para títulos convencionais que não têm entrada subordinada a no-
mes. O campo 840 continha os seguintes indicadores: $1^{\circ}$ indicador:
indefinido (\#), $2^{\circ}$ indicador: $0-9$ (número de caracteres a desprezar).
Os subcampos eram $\$ a$ (Título), $\$$ h (Meio [DGM]) e \$v (Volume
ou número).

${ }^{1}$ O campo 840 tornou-se obsoleto em 1980 . A partir de então de-
cidiu-se utilizar o campo 830 tanto para títulos uniformes quanto
para títulos convencionais que não têm entrada subordinada a no-
mes. O campo 840 continha os seguintes indicadores: $1^{\circ}$ indicador:
indefinido (\#), $2^{\circ}$ indicador: $0-9$ (número de caracteres a desprezar).
Os subcampos eram $\$ a$ (Título), $\$$ h (Meio [DGM]) e \$v (Volume
ou número).

${ }^{1}$ O campo 840 tornou-se obsoleto em 1980 . A partir de então de-
cidiu-se utilizar o campo 830 tanto para títulos uniformes quanto
para títulos convencionais que não têm entrada subordinada a no-
mes. O campo 840 continha os seguintes indicadores: $1^{\circ}$ indicador:
indefinido (\#), $2^{\circ}$ indicador: $0-9$ (número de caracteres a desprezar).
Os subcampos eram $\$ a$ (Título), $\$$ h (Meio [DGM]) e \$v (Volume
ou número).
} Ci. Inf., Brasília, DF, v. 39 n. 3, p.47-60, set./dez., 2010 
Aplicabilidade dos campos 490 e 800-830 do Formato MARC 21 para Dados Bibliográficos

Indicadores:

1 Tipo de entrada do nome pessoal:

0 Prenome;

1 Sobrenome;

3 Nome de família.

2 Indefinido (\#).

Subcampos:

\$a Nome pessoal (NR);

\$b Numeração (algarismos romanos que seguem o prenome) (NR);

\$c Títulos e outras palavras associadas ao nome (NR);

\$d Datas associadas ao nome (NR);

\$e Termo relacionador (R);

\$f Data da publicação (NR);

\$g Informações adicionais(NR);

\$h Meio (DGM) (NR);

\$j Atributo (R);

\$k Subcabeçalho de forma (R);

\$1 Idioma da publicação (NR);

\$m Instrumento musical (R);

\$n Número da parte/seção da publicação (R);

\$o Arranjo musical (NR);

\$p Nome da parte/seção da publicação (R);

\$q Forma completa do nome (NR);

\$r Escala musical (NR);

\$s Versão (NR);

\$t Título da publicação (NR);

\$u Afiliação (NR);

Ci. Inf., Brasília, DF, v. 39 n. 3, p.47-60, set./dez., 2010 \$v Volume ou número sequencial da série (NR);

\$w Número de controle do registro bibliográfico $(\mathrm{R})$;

\$x ISSN (NR);

\$0 Número de controle do registro de autoridade (R);

\$3 Especificação do material (NR);

\$4 Código de termo relacionador $(\mathrm{R})$;

\$5 Instituição (R);

\$6 Ligação (NR);

\$8 Campo de ligação e número sequencial (R).

Para a aplicação do campo 800 faz-se necessário o cadastro da autoridade 100 - Heading - Personal Name (Cabeçalho - Nome pessoal) do MARC 21 Autoridade. Dessa forma, cabe aqui a descrição desse campo e a finalidade de seus elementos.

\section{Cabeçalho - Nome Pessoal}

O presente trabalho focaliza a utilização do campo 100 do MARC 21 Autoridade para uso no campo 800 do MARC 21 Bibliográfico. Essa definição faz-se necessária tendo em vista que os elementos utilizados para o registro de uma autoridade do campo 100, objetivando seu uso nos campos 100 (Entrada Principal - Nome pessoal), 600 (Assunto - Nome pessoal) e 700 (Entrada secundária - Nome pessoal) do MARC 21 Bibliográfico são diferentes dos elementos utilizados no campo 800 - Entrada secundária de série - Nome pessoal e exigem cadastros separados na base de autoridades.

Os elementos que compõem o campo 100 são os seguintes:

Indicadores:

1 Tipo de entrada do nomes pessoal:

0 Prenome;

1 Sobrenome; 
3 Nome de família.

2 Indefinido (\#).

Subcampos:

\$a Nome pessoal (NR);

\$b Numeração (algarismos romanos que seguem o prenome) (NR);

\$c Títulos e outras palavras associadas ao nome (NR);

\$d Datas associadas ao nome (NR);

\$e Termo relacionador $(\mathrm{R})$;

\$f Data da publicação (NR);

\$g Informações adicionais(NR);

\$h Meio (DGM) (NR);

\$j Atributo (R);

\$k Subcabeçalho de forma (R);

\$1 Idioma da publicação (NR);

\$m Instrumento musical (R);

\$n Número da parte/seção da publicação (R);

\$o Arranjo musical (NR);

\$p Nome da parte/seção da publicação (R);

\$q Forma completa do nome (NR);

\$r Escala musical (NR);

\$s Versão (NR);

\$t Título da publicação (NR);

\$v Subdivisão de forma (R);

\$x Subdivisão geral (R);

\$y Subdivisão cronológica (R);

\$z Subdivisão geográfica (R);

\$6 Ligação (NR);

\$8 Campo de ligação e número sequencial (R).
Com os dados anteriormente dispostos, conclui-se que para o preenchimento do campo 100 para uso no campo 800 deve-se trabalhar com os elementos relacionados ao nome e ao título. Por indicação do Conselho Consultivo do MARC, tais elementos devem seguir as orientações do AACR2 e o Library of Congress Subject Headings - LCSH (Cabeçalhos de Autoridades da Biblioteca do Congresso)

No que se refere às regras do AACR2 a serem utilizadas, iremos detalhar apenas as que ditam o uso dos subcampos k e t. Para melhor compreensão, observe os exemplos seguintes:

Título da série na obra: Obras completas de Machado de Assis

no registro bibliográfico:

4901 \# \$a Obras completas de Machado de Assis; \$v 5; 8001 \# \$a Assis, Machado, \$d 1888-1999. \$t Obras; \$v 5 .

no registro de autoridade:

1001 1\# \$a Assis, Machado, \$d 1888-1999. \$t Obras;

400 1\# \$a Assis, Machado, \$d 1888-1999. \$t Obras completas de Machado de Assis;

430 \#0 \$a Obras completas de Machado de Assis².

O subcampo t foi elaborado de acordo com a regra 25.8A do AACR2 que diz o seguinte:

Use o título coletivo Obras para um item que consiste das obras completas de uma pessoa, ou que é apresentado como tal, incluindo obras completas na época da publicação.

Mas supondo que o título da série fosse "Obras de Machado de Assis" (confirmando não ser a coleção completa, mas três ou mais obras do autor) o registro da série no campo 100 seria feito da seguinte forma:

${ }^{2}$ No Formato MARC 21 para dados de Autoridade os campos 4XX indicam formas remissivas da entrada autorizada.

Ci. Inf., Brasília, DF, v. 39 n. 3, p.47-60, set./dez., 2010 
Aplicabilidade dos campos 490 e 800-830 do Formato MARC 21 para Dados Bibliográficos

100 1\# \$a Assis, Machado, \$d 1888-1999. \$t Seleções;

400 1\# \$a Assis, Machado, \$d 1888-1999. \$t Obras de Machado de Assis;

430 \#0 \$a Obras de Machado de Assis.

Nesse caso, utilizou-se a regra 25.9A:

Use o título coletivo Seleções para itens que consistem de três ou mais obras em várias formas, ou em uma única forma, se a pessoa tiver produzido suas obras somente de uma forma, e para itens que consistem de excertos etc. das obras de uma só pessoa. Para obras musicais, veja também 25.34B-25.34C.

Os campos 4XX são utilizados seguindo a regra 26.5A1, que diz o seguinte:

Se uma entrada secundária for feita sob o cabeçalho de uma série à qual pertencem partes catalogadas separadamente, faça remissivas para o cabeçalho da série das diferentes formas do cabeçalho sob o qual poderia ser razoavelmente procurado.

Quando se trata de obras completas de uma pessoa e a obra apresenta uma forma específica, segue-se a regra 25.10, utilizando-se os seguintes títulos coletivos:

Contos;

Correspondência;

Ensaios;

Romances;

Discursos;

Poemas;

Obras em Prosa;

Peças de teatro.

O AACR2 permite ainda que se elaborem títulos coletivos específicos, caso nenhum dos termos citados seja apropriado para representar o título coletivo da obra que se está catalogando.

Para os casos em que a série consiste de três ou mais obras, mas não a coleção completa, e se trata de um título coletivo específico, deve-se acrescentar a palavra "Seleções" no subcampo k, conforme exemplo:

Título da série na obra: Teatro de Millôr Fernandes no registro bibliográfico:

490 1\# \$a Teatro de Millôr Fernandes ; \$v 3;

8001 \$a Fernandes, Millôr, \$d 1924-. \$t Peças de Teatro. \$k Seleções ; \$v 3.

no registro de autoridade:

1001 \$a Fernandes, Millôr, \$d 1924-. \$t Peças de Teatro. \$k Seleções;

4001 \$a Fernandes, Millôr, \$d 1924-. \$t Teatro de Millôr Fernandes;

4301 \$a Teatro de Millôr Fernandes.

\section{Entrada secundária de série - Entidade coletiva}

O campo 810 é definido como uma entrada secundária de série na qual a entrada de autor é uma entidade coletiva. É utilizado sempre que a obra descrita tiver sua entrada principal dada por uma entidade (ver regras 21.1B2 e 21.6C2 do AACR2 ).

Assim como o campo 800, o uso do campo 810 geralmente se justifica pela presença de um 490 (Título de série) ou um 500 (Notas gerais) relativo à série. Para reproduções, poderá ainda ser justificado por um título de série no subcampo $\$$ f do campo 533 (Notas de reprodução).

O campo 810 é repetitivo (R) e inclui os seguintes elementos:

Indicadores:

1 Tipo de entrada do nome da entidade:

0 Nome invertido;

1 Nome da jurisdição (lugar); 
3 Nome na ordem direta.

2 Indefinido (\#).

Subcampos:

\$a Nome da entidade ou da jurisdição (lugar) pela qual será feita a entrada (NR);

\$b Unidade subordinada (R);

\$c Local de realização do evento (NR);

\$d Data de realização do evento ou assinatura do tratado $(\mathrm{R})$;

\$e Termo relacionador $(\mathrm{R})$;

\$f Data da publicação (NR);

\$g Informações adicionais (NR);

\$h Meio (DGM) (NR);

\$k Subcabeçalho de forma (R);

\$1 Idioma da publicação (NR);

\$m Instrumento musical (R);

\$n Número da parte/seção/evento (R);

\$o Arranjo musical (NR);

\$p Nome da parte/seção da publicação (R);

\$r Escala musical (NR);

\$s Versão (NR);

\$t Título da publicação (NR);

\$u Afiliação (NR);

\$v Volume/Designação sequencial (R);

\$w Número de controle do registro bibliográfico (R);

\$x ISSN (NR);

\$0 Número de controle do registro de autoridade $(\mathrm{R})$;

\$3 Especificação do material (NR);

$\$ 4$ Código do termo relacionador $(\mathrm{R})$; \$5 Instituição (R);
\$6 Ligação (NR);

\$8 Campo de ligação e número sequencial (R).

Para a aplicação do campo 810, faz-se necessário o cadastro da autoridade 110 - Heading - Corporate name (Cabeçalho - Entidade coletiva) do MARC 21 Autoridade. Como foi explicado no item anterior, para o preenchimento do campo 110 (autoridade) que será utilizado no campo 810 (bibliográfico) deve-se trabalhar com os elementos relacionados ao nome da entidade e ao título. Por indicação do Conselho Consultivo do MARC, tais elementos devem seguir as orientações do AACR2 e do LCSH.

Para melhor compreensão, observe os exemplos a seguir:

Título da série na obra: CILT linguistic atlas service no registro bibliográfico:

4901 \# \$a CIL linguistic atlas series ; \$v 2;

$8102 \#$ \$a Central Institute of Indian Languages. \$t CIL linguistic atlas series ; \$v 2.

no registro de autoridade:

110 2\# \$a Central Institute of Indian Languages. \$t CIL linguistic atlas series;

430 \#0 \$a CIL linguistic atlas series.

No exemplo anterior, o título da série ficou subordinado à entidade devido à participação desta na autoria da obra. Caso a entidade não tivesse a responsabilidade principal sobre o conteúdo da obra, a entrada secundária para o título de série deveria ter sido feita diretamente no campo 830.

\section{Entrada secundária de série - Evento}

O campo 811 é definido como uma entrada secundária de série na qual a entrada de autor é um evento. É utilizado sempre que a obra descrita tiver sua entrada por conferências, congressos, reuniões etc. (ver regras 24.7 e $21.6 \mathrm{C} 2$ do AACR2) 
O uso do campo 811 geralmente se justifica pela presença de um 490 (Título de série), ou um 500 (Notas gerais) relativo à série. Para reproduções, poderá ainda ser justificado por um título de série no subcampo \$f do campo 533 (Notas de reprodução). Entradas secundárias de série para eventos cuja entrada principal é subordinada a uma entidade coletiva deverão ser registradas no campo 810.

O campo 811 é repetitivo (R) e inclui os seguintes elementos:

Indicadores:

1 Tipo de entrada do nome do evento:

0 Nome invertido;

1 Nome da jurisdição (lugar);

3 Nome na ordem direta.

2 Indefinido (\#).

Subcampos:

\$a Nome do evento ou da jurisdição (lugar) pela qual será feita a entrada (NR);

\$c Local de realização do evento (NR);

\$d Data de realização do evento (NR);

\$e Unidades subordinadas (R);

\$f Data da publicação (NR);

\$g Informações adicionais (NR);

\$h Meio (DGM) (NR);

$\$ \mathrm{j}$ Termo relacionador $(\mathrm{R})$;

\$k Subcabeçalho de forma (R);

\$1 Idioma da publicação (NR);

\$n Número da parte/seção/evento (R);

\$p Nome da parte/seção da publicação (R);

\$q Nome do evento seguido do nome da jurisdição (NR);

Ci. Inf., Brasília, DF, v. 39 n. 3, p.47-60, set./dez., 2010 \$s Versão (NR);

\$t Título da publicação (NR);

\$u Afiliação (NR);

\$v Volume/Designação sequencial (R);

\$w Número de controle do registro bibliográfico (R);

\$x ISSN (NR);

\$0 Número de controle do registro de autoridade $(\mathrm{R})$; \$3 Especificação do material (NR); $\$ 4$ Código do termo relacionador (R); \$5 Instituição (R);

\$6 Ligação (NR);

\$8 Campo de ligação e número sequencial (R).

Para a aplicação devida do campo 811, faz-se necessário o cadastro da autoridade 111 -Heading - Meeting name (Cabeçalho - Evento) do MARC 21 Autoridade. Como foi explicado nos itens anteriores, para o preenchimento do campo 111 (autoridade) que será utilizado no campo 811 (bibliográfico) deve-se trabalhar com os elementos relacionados ao nome do evento e ao título. Por indicação do Conselho Consultivo do MARC tais elementos devem seguir as orientações do AACR2 e do LCSH.

Para melhor compreensão, observe os exemplos a seguir:

Título da série na obra: Nutrition and food science 490 1\# \$a Nutrition on food science;

811 2\# \$a International Congress of Nutrition. \$n (11. : \$d 1978 : \$c Rio de Janeiro/RJ). \$t Nutrition and food science.

no registro de autoridade:

1112 \# \$a International Congress of Nutrition. \$n (11. : \$d 1978 : \$c Rio de Janeiro/RJ). \$t Nutrition and food science; 
411 2\# \$a International Congress of Nutrition. \$t Nutrition and food science;

430 \#0 \$a Nutrition and food science.

No exemplo anterior, o título da série ficou subordinado ao evento devido à participação deste na autoria da obra. Caso o evento não tivesse a responsabilidade principal sobre o conteúdo da obra, a entrada secundária para o título de série deveria ter sido feita diretamente no campo 830.

\section{Entrada secundária de série - Título} uniforme

O campo 830 é definido como uma entrada secundária de título de série. É utilizado sempre que o título de série descrito no campo 490 difere da forma controlada.

Assim como os campos 800, 810 e 811 , o uso do campo 830 geralmente se justifica pela presença de um 490 (Título de série) ou um 500 (Notas gerais) relativo à série. Para reproduções, poderá ainda ser justificado por um título de série no subcampo $\$ \mathrm{f}$ do campo 533 (Notas de reprodução).

O campo 830 é repetitivo (R) e inclui os seguintes elementos:

Indicadores:

1 Indefinido (\#);

2 Número de caracteres a desprezar (0-9).

Subcampos:

\$a Título uniforme (NR);

\$d Data de assinatura do tratado (R);

\$f Data da publicação (NR);

\$g Informações adicionais (NR);

\$h Meio (DGM) (NR);

\$k Subcabeçalho de forma (R);

\$m Instrumentos musicais $(\mathrm{R})$; \$n Número da parte/seção/evento (R);

\$o Arranjo musical (NR);

\$p Nome da parte/seção da publicação (R);

\$r Escala musical (R);

\$s Versão (NR);

\$t Título da publicação (NR);

\$v Volume/Designação sequencial (R);

\$w Número de controle do registro bibliográfico (R);

\$x ISSN (NR);

\$0 Número de controle do registro de autoridade $(\mathrm{R})$; \$3 Especificação do material (NR); \$5 Instituição (R);

\$6 Ligação (NR);

\$8 Campo de ligação e número sequencial (R).

Para a aplicação do campo 830, faz-se necessário o cadastro da autoridade 130 - Heading - Uniform title (Cabeçalho - Título uniforme) do MARC 21 Autoridade.

Para melhor compreensão, observe os exemplos a seguir:

a) Título da série na obra: Basic paper

no registro bibliográfico:

490 1\# \$a Basic paper; \$v 1983, n. 5;

830 \#0 Basic paper (Australia. Department of the Parliamentary Library. Legislative Research Service) ; \$v 1983, n. 5.

no registro de autoridade:

130 \#0 \$a Basic paper (Australia. Department of the Parliamentary Library. Legislative Research Service);

41010 \$a Australia. \$b Department of the Parliamentary Library. \$b Legislative Research Service. \$t Basic paper. 
Aplicabilidade dos campos 490 e 800-830 do Formato MARC 21 para Dados Bibliográficos

b) Título da série na obra: Inaugural lecture

no registro bibliográfico:

4901 \# \$a Inaugural lecture / University of Cape Town, new series ; \$v 170;

830 \#0 \$a Inaugural lecture (University of Cape Town). \$n New series; \$ 170.

no registro de autoridade:

130 \#0 \$a Inaugural lecture (University of Cape Town). \$n New series;

410 2\# \$a University of Cape Town. \$t Inaugural lecture. $\$ \mathrm{n}$ New series;

430 \#0 \$a Inaugural lecture. \$n New series.

Nos exemplos "a" e "b", a forma padronizada da série recebeu uma indicação de responsabilidade de acordo com a regra 1.6E1 do AACR2.

No exemplo "b" há, ainda, a presença do subcampo \$n para o registro da informação de nova série. De acordo com a regra $1.6 \mathrm{H} 3$ do AACR2,

se aparecer uma frase com "nova série", "segunda série" etc. como uma série não numerada, transcreva essa frase com o título da subsérie [subcampo \$p]. Se aparecer como uma série numerada, transcreva a frase como parte da numeração da série [subcampo $\$ \mathrm{n}]$, de acordo com as instruções de 1.6G1 (veja também 12.3G).

No caso de dúvida, se um dos títulos mencionados é uma subsérie ou uma segunda série, a regra 1.6H4 recomenda tratar o título como uma segunda série (veja o exemplo a seguir).

c) Título da série na obra: Biblioteca do Exército, v. 526. Coleção General Benício, v. 210

no registro bibliográfico:

4901 \# \$a Biblioteca do Exército ; \$v 526. \$a Coleção General Benício ; \$v 210.
No campo 490, quando uma subsérie é separada da série principal por uma numeração ou quando a série tem um título paralelo, o subcampo \$a deverá ser repetido.

830 \#0 \$a Biblioteca do Exército; \$v 526;

830 \#0 \$a Coleção General Benício ; \$v 210.

A indicação de série contém uma série numerada e uma subsérie e ambas deverão ser desdobradas separadamente.

No registro de autoridade (entradas independentes):

130 \#0 \$a Biblioteca do Exército;

130 \#0 \$a Coleção General Benício.

O exemplo "c" foi elaborado conforme orientações da regra 1.6J do AACR2.

\section{CONSIDERAÇÕES FINAIS}

A utilização do formato MARC pelos sistemas de gerenciamento de bibliotecas é de fundamental importância para a construção de formas de representação para os recursos informacionais e para o intercâmbio de dados bibliográficos, possibilitando a interoperabilidade entre sistemas de informação.

Ao longo das pesquisas realizadas, por diversas vezes percebeu-se a ausência de bibliografia sobre MARC em língua portuguesa. Chamou a atenção também a ausência de dúvida por parte dos catalogadores brasileiros perante as modificações realizadas em uma das principais áreas da catalogação: a Série. Poucos profissionais manifestaram-se para explicar o que as mudanças provocariam na representação dos conteúdos e os benefícios que tanto os profissionais quanto os usuários teriam.

No Brasil, o formato MARC é visto como um qualificador de bibliotecas e é comum ver em apresentações institucionais as expressões "Trabalhamos com o formato MARC" ou "Nossa 
catalogação segue o padrão MARC”. Mas é esse o principal questionamento que levantamos sobre a catalogação brasileira: ela apenas "segue o padrão MARC" (entenda aqui a expressão "seguir" como "estar sempre atrás"), já que as bibliotecas brasileiras nunca acompanham efetivamente o que está sendo decidido acerca desse conglomerado de campos, subcampos e indicadores que constituem o MARC 21.

Sobre a extinção do campo 440 em favor dos campos 490 e 800-830, acreditamos que foi uma decisão acertada e apresenta suas vantagens:

- elimina a possibilidade de duplicidade de registros no campo da série como acontecia com os campos 440 e 490;

- melhora a indexação de entradas secundárias de série, alterando o fluxo de trabalho do catalogador e reforçando a necessidade de controle da base de autoridades;

- proporciona uma descrição confiável dentro dos padrões de catalogação e de fácil manutenção ao longo do tempo.

Porém, como qualquer mudança acarreta uma série de consequências, como as seguintes:

- a impossibilidade de conversão automática dos campos 440 em 490;

- a presença de informações em um campo obsoleto (campo 440) durante um período indeterminado ou até que se revise todo o catálogo;

- a necessidade de melhor preparo e maior conhecimento técnico do catalogador sobre as regras que regem a área da série.

Como já mencionado anteriormente, observa-se a ausência de posicionamento das entidades nacionais responsáveis pela catalogação diante das alterações realizadas no formato MARC e que diretamente ativam regras do AACR2 que, até então, não eram utilizadas ou que não recebiam destaque. Nesse ponto, parece-nos claro que muitas instituições preferem pagar por uma base de autoridades, terceirizando o seu trabalho intelectual, em lugar de investir na qualificação de suas equipes para que essas possam gerir com autonomia suas bases bibliográficas.

Assim, o trabalho apresentado buscou elucidar questões que, até o presente, não estão sendo tratadas na biblioteconomia brasileira, e explicar aos profissionais bibliotecários, principalmente os que atuam em setores de processamento técnico, o que muda com a alteração aprovada em 2009 e o impacto dessa mudança no processo de catalogação.

A catalogação brasileira ainda precisará acompanhar diversas mudanças, haja vista a revisão do AACR2, sua extinção em favor da Resource Description and Access - RDA e uma série de alterações no formato MARC em função desse novo código de catalogação. O ideal seria que houvesse maior envolvimento por parte das instituições e profissionais brasileiros no acompanhamento e discussão dessas mudanças.

Artigo submetido em 04/08/2010 e aceito em 29/06/2011.

\section{REFERÊNCIAS}

CÓDIGO de catalogação anglo-americano. 2. ed. rev. São Paulo: FEBAB ; Imprensa Oficial do Estado, 2004.

LIBRARY OF CONGRESS. Network Development and MARC Standards Office. MARC discussion paper no. 2008-DP02: making field 440 (Series Statement/Added Entry-Title) obsolete in the MARC 21 Bibliographic Format. 2007. Disponível em: <http://www.loc.gov/ marc/marbi/2008/2008-dp02.html>. Acesso em: jun. 2010.

. MARC proposal no. 2008-06 : adding information associated with the Series Added Entry fields (800-830). 2008. Disponível em: <http://www.loc.gov/marc/marbi/2008/2008-06.html>. Acesso em: jun. 2010.

MARC proposal no. 2008-07 : making field 440 (Series Statement/Added Entry--Title) obsolete in the MARC 21 Bibliographic Format. 2008. Disponível em: <http://www.loc.gov/ marc/marbi/2008/2008-07.html>. Acesso em: jun. 2010.

. MARC 21 Format for Authority Data. 1999 edition. Disponível em: <http://www.loc.gov/marc/authority/ecadhome.html > Acesso em: jun. 2010.

MARC 21 Format for Bibliographic Data. 1999 edition. Disponível em: <http://www.loc.gov/marc/bibliographic/ ecbdhome.html>. Acesso em: jun. 2010. 